\title{
Non-Gaussian amplitude PDF modeling of ultrasound images based on a novel generalized Cauchy-Rayleigh mixture
}

\author{
Mohammadreza Hassannejad Bibalan and Hamidreza Amindavar ${ }^{*}$
}

\begin{abstract}
In this paper, a mixture of generalized Cauchy distribution and Rayleigh distribution that possesses a closed-form expression is proposed for modeling the heavy-tailed Rayleigh (HTR) distribution. This new approach is developed for analytically modeling the amplitude distribution of ultrasound images based on the HTR distribution. HTR as a non-Gaussian distribution is basically the amplitude probability density function (PDF) of the complex isotropic symmetric $\alpha$-stable $(S \alpha S)$ distribution which appears in the envelope distribution of ultrasonic images. Analytic expression for HTR distribution is a momentous consideration in signal processing with stable random variables. Furthermore, we introduce a mixture ratio estimator based on the energy of amplitude PDF which contains both $\alpha$ and $\gamma$ parameters. For a quantitative assessment, we compare the accuracy and computational complexity of the proposed mixture with other approximations of HTR distribution through several numeral simulations on synthetic random samples. Experimental results obtained from the Kolmogorov-Smirnov (K-S) distance and Kullback-Leibler (K-L) divergence as the goodness-of-fit tests on real ultrasound images reveal the favor of the new mixture model.
\end{abstract}

Keywords: Generalized Cauchy-Rayleigh model, Heavy-tailed Rayleigh distribution, $\alpha$-stable distribution, Non-Gaussian amplitude PDF, Ultrasound images

\section{Introduction}

In the medical context, ultrasound provides a noninvasive technique of imaging human anatomy with good visualization characteristics and relatively easy management [1]. As a widely used medical imaging modality, ultrasound applications include cardiology, urology, obstetrics and gynecology, general abdominal imaging, vascular imaging, ophthalmology, orthopedics, and surgical procedures [2,3]. B-mode (brightness mode) has been the widely accepted method for ultrasound imagery. In this mode of ultrasound, a linear array of transducers simultaneously scans a plane through the body that can be viewed as a 2D image on screen [4]. Modeling the distribution statistics of ultrasound images has its own importance and strongly depends on a comprehensive knowledge of tissue scattering mechanism [5]. In recent years, there has been a growing interest in modeling the amplitude probability density function

*Correspondence: hamidami@aut.ac.ir

Amirkabir University of Technology, Tehran, Iran
(PDF) of ultrasound images specifically in speckle denoising [6].

Several models have been utilized to statistically characterize the envelope distribution of ultrasound returns [7]. Three major categories used in the amplitude PDF estimation of ultrasound images are summarized into parametric, nonparametric, and mixture models [8]. The most used models for this purpose are the Rayleigh model [9], K-distribution [10], Nakagami distribution [11], and generalized Nakagami distribution [12]. In addition to these methods, one can directly use the return data to construct the amplitude distribution of ultrasound images, for example, the heavy-tailed Rayleigh (HTR) distribution. The HTR distribution arises naturally in scenarios involving scattering effects with scatterers with crosssection distributions that are heavy tailed, and it has found applications in many domains including ultrasound imaging and SAR. Specifically, the HTR model is considered as the most theoretically well-founded statistical model at present. The HTR distribution can model 
many classes of ultrasound images [13]. The main consideration in signal processing with HTR distribution is having no closed-form expression for its statistics. In other words, there is no analytic formula for the PDF of the HTR distribution. One solution to overcome this limitation is utilizing the mixture approximation. This drawback also makes it difficult to estimate its parameters (it is difficult to derive the maximum likelihood (ML) estimators). We propose a new approximation with a tractable density, with a novel estimator for its mixture ratio.

For the HTR distribution to be practically used for any ultrasound imagery application, one must be competent to estimate the parameters characteristic exponent, $\alpha$, and dispersion, $\gamma$, from the observed data. The ML estimate can be obtained by letting the derivatives of the loglikelihood function to zero and solving the resultant transcendental equations. Although, it is time consuming and is not an effective procedure. Moreover, the most important disadvantage of this method is having no explicit expressions for parameter solutions. In the method of moments (MOM), only the negative-order moments of the distribution were exploited to estimate the parameters [14]. The method of log-cumulants (MOLC) is an extension of MOM, by utilizing the Mellin transform (MT) in place of the usual Fourier and Laplace transforms in statistical computations [15]. Still by analogy with classical statistic for scalar real random variables defined in $\mathbb{R}^{+}$, the second characteristic function (CF) of the second kind is defined as the natural logarithm of the first CF of the second kind. Parameter estimation based on the MT is a high accuracy and precision method to extracting the statistical features of ultrasound images.

A typical problem in ultrasound signal processing for coherent image formation is that the tissue scatterer is influenced by multiplicative speckle noise [16]. The multiplicative model was proposed for describing the statistical properties of the ultrasound returns. According to the multiplicative model, several PDFs are developed such as log-normal distribution, K-distribution, and G-distribution. K-distribution is a particular form of the $\mathrm{G}$ model, which assumes both the tissue and the speckle component as gamma distribution [17]. Rician inverse Gaussian (RiIG) distribution is derived under the assumption that the scattering process acts as a Wiener Brownian motion with drift, superimposed on an inverse Gaussian (IG) distributed stopping time [18]. In many respects, this model is similar to the $\mathrm{K}$ model, but it has a flexible parameterization, which makes it more versatile.

Through this paper, we statistically model the envelope distribution of ultrasound images as a new generalized Cauchy-Rayleigh mixture approximation based on HTR distribution. Moreover, analytical derivation for mixture ratio estimation based on the characteristic exponent parameter and the dispersion parameter of HTR distribution which has closed-form expression is derived.

The rest of this paper is organized as follows. Section 2 provides the problem statement for describing the statistics behavior of ultrasound images and related parameter estimation methods. Specifically, three major mixture approximations of HTR distribution are given. In Section 3, our novel mixture approximation for HTR distribution is proposed and derivation of the mixture ratio estimator is provided. We also evaluate the performance of our proposed model through numerical simulations in Section 4. Furthermore, Section 5 provides experiments on real ultrasound images of different kinds. Finally, the paper is concluded in Section 6.

\section{Amplitude statistics of ultrasound images}

In this section, the prominent models used for modeling the amplitude PDF of ultrasound images are examined. We consider the RiIG model, the $\mathrm{K}$ model, and the HTR model with their parameter estimation methods.

\subsection{RilG distribution}

A mixture of the Rice distribution [19] and the inverse Gaussian distribution homonymous to the Rician inverse Gaussian (RiIG) distribution has been addressed as a function of three parameters for amplitude statistics of ultrasonic images [18]. This model introduces a new compound statistical model for modeling non-Rayleigh amplitude signals. The RiIG PDF is given by

$$
f_{\mathrm{r}}(r)=\sqrt{\frac{2}{\pi}} a^{\frac{3}{2}} \delta e^{\delta \lambda} \frac{r}{\left(r^{2}+\delta^{2}\right)^{\frac{3}{4}}} K_{\frac{3}{2}}\left(a \sqrt{r^{2}+\delta^{2}}\right) I_{0}(b r),
$$

where $\lambda=\sqrt{a^{2}-b^{2}}, K_{\frac{3}{2}}(\cdot)$ is the modified Bessel function of the second kind and $I_{0}(\cdot)$ denotes the modified Bessel function of the first kind. It can be seen from (1) that the model has three parameters and it has capability to cover a wide range of shapes. Parameter estimation of the RilG model is performed based on the iterative maximum likelihood method (IMLM). Let $\left\{r_{k}\right\}, k=$ $1,2, \cdots, N$, denote a set of independent observations of RiIG-distributed random variables. For some initial values for $a_{0}$ and $\delta_{0}$ and letting $v_{k}=\sqrt{r_{k}^{2}+\delta_{0}^{2}}, \hat{\mu}$ may then be estimated from the ultrasound data as the following:

$$
\hat{\mu}=\frac{1}{N} \sum_{k=1}^{N}\left(\frac{v_{k}}{a_{0}} \frac{v_{k} a_{0}}{1+v_{k} a_{0}}\right),
$$

and the estimate of $\hat{\mu}^{-1}$ is expressed as 


$$
\hat{\mu}^{-1}=\frac{1}{N} \sum_{k=1}^{N}\left(\frac{a_{0}}{v_{k}} \frac{\left(v_{k} a_{0}\right)^{2}+3 v_{k} a_{0}+3}{v_{k} a_{0}\left(1+v_{k} a_{0}\right)}\right) .
$$

Using (2) and (3), the RiIG estimators for $\delta$ and $\gamma$ become $\hat{\delta}=\sqrt{\frac{1}{\hat{\mu}^{-1}}-\frac{1}{\hat{\mu}}}$ and $\hat{\gamma}=\frac{\hat{\delta}}{\hat{\mu}}$, respectively. A new estimate for $\hat{a}$ may be obtained using the ML method which is accordingly given as $\hat{a}=\arg \max F(\hat{a} ; \hat{\delta}, \hat{\lambda}, \boldsymbol{r})$, where,

$$
F(\hat{a} ; \hat{\delta}, \hat{\lambda}, \boldsymbol{r})=\ln \prod_{k=1}^{N} f_{\mathrm{r}}\left(r_{k}\right)=\sum_{k=1}^{N} \ln f_{\mathrm{r}}\left(r_{k}\right) .
$$

The exact solution to this optimization must be numerically accomplished. The aforementioned steps should continue till a maximum number of iterations has been reached or till the desired convergence is achieved. From another point of view, the RiIG distribution has similarity to the $\mathrm{K}$ model; whereas its parameter estimation procedure is more computationally expensive, RiIG posterior distribution can be expressed in analytic form, which makes it facile to establish a maximum a posteriori (MAP) speckle filter.

\subsection{K-Distribution}

K-Distribution is the most famous distribution for modeling the amplitude PDF of non-Rayleigh ultrasound which was first proposed to describe the spatial distribution of certain larvae in terms of a two-dimensional (2D) Brownian motion model, coupled with an exponential birth-death distribution [20]. In other words, the number of scatterers from the tissue is in itself a random variable and the population of scatterers is controlled by a birth-death-migration process. Unlike the RiIG model, the K-distribution is a two-parameter model. The corresponding amplitude distribution of the ultrasound image based on the K-distribution is defined by

$$
f_{\mathrm{r}}(r)=\frac{2}{\sigma \Gamma(v+1)}\left(\frac{r}{2 \sigma}\right)^{\nu+1} K_{v}\left(\frac{r}{\sigma}\right)
$$

where $K_{v}(\cdot)$ is the modified Bessel function of the second kind. Also for the K-distribution, the parameter estimation can be done by the solution of the following equations [21]:

$$
\begin{aligned}
& \psi(1, v+1)-4 \hat{\tilde{k}}_{2}+\psi(1,1)=0, \\
& \hat{\sigma}=\frac{1}{2} \exp \left\{\hat{\tilde{k}}_{1}-\frac{\psi(\hat{v}+1)+\psi(1)}{2}\right\},
\end{aligned}
$$

where $\tilde{k}_{n}$ denotes the log-cumulant of degree $n$.

\subsection{HTR distribution}

PDF for $\alpha$-stable random variate is provided by taking the inverse Fourier transform of its characteristic function (CF) [22]. Suppose $-\infty<x<\infty$ and $x \sim S(\alpha, \gamma, \beta, \mu)$, its PDF is completely determined by four parameters; however, a closed-form formula does not exist for its PDF [23]. $\alpha$ is the characteristic exponent and it determines the shape of the distribution $(0<\alpha \leq 2), \gamma$ is the dispersion or scale parameter of the distribution and plays a similar role to the variance of the Gaussian distribution $(\gamma>0), \beta$ is the index of skewness $(-1 \leq \beta \leq 1)$, and $\mu$ is the location parameter $(\mu \in \mathbb{R})$. The case $\beta=0$ corresponds to the symmetric $\alpha$-stable distribution $(S \alpha S)$. A complex random variable $x=x_{i}+j x_{q}=r e^{j \phi}$ is $S \alpha S$ if $x_{i}$ and $x_{q}$ are jointly $S \alpha S$ and symmetric. So $x$ is a symmetric bivariate $\alpha$-stable r.v. The PDF of $x_{i}$ and $x_{q}$ is $S \alpha S$ distributed; furthermore, the absolute PDF of $x$ is distributed according to a HTR distribution [13]. In [24], a new method was proposed to generalize the Gaussian model for the complex received ultrasound signal by assuming both real and imaginary components to be distributed according to an $\alpha$-stable distribution. The backscattered ultrasound RF signals establish a stable process which has non-Gaussian $\alpha$-stable limit distribution, and the envelope of the backscattered signal is shown to follow a HTR distribution. This HTR model has more flexibility than the classical Rayleigh model by considering the impulsive behavior of ultrasound images [14]. Hereinafter, we focus on HTR distribution with $1 \leq \alpha \leq 2$, which is defined in terms of its CF, as the following:

$$
\varphi_{\alpha, \gamma}(\omega)=\exp \left(-\gamma|\omega|^{\alpha}\right)
$$

where, $\boldsymbol{\omega}=\left(\omega_{1}, \omega_{2}\right)=\omega_{1}+j \omega_{2}$. In the literature [25], the HTR distribution is defined as

$$
f_{\alpha, \gamma}(r)=r \int_{0}^{\infty} \omega \exp \left(-\gamma \omega^{\alpha}\right) J_{0}(r \omega) d \omega
$$

where $r$ is the amplitude and $J_{0}(\cdot)$ is the Bessel function of the first kind. The PDF characterized by (9) is demonstrated as an empirical model which is ideal for modeling ultrasound amplitude RF returns. The reason for this appellation is that this new generalized form of the Rayleigh distribution can illustrate impulsive behavior and has heavier tails rather than the classical Rayleigh distribution. Therefore, the noise-free ultrasound return signal amplitude PDF can be modeled by HTR distribution. Since the HTR distribution assumes the zero probability at the origin $(f(r)=0$ at $r=0)$, the ultrasound images should have no pixel with zero intensity (black margins). An analytic formula only exists for two special values of $\alpha$. The case $\alpha=2$ is the following:

$$
f_{2, \gamma}(r)=\frac{r}{2 \gamma} \exp \left(-\frac{r^{2}}{4 \gamma}\right),
$$


which is basically the classical Rayleigh distribution. The other special case is $\alpha=1$ :

$$
f_{1, \gamma}(r)=\frac{\gamma r}{\left(r^{2}+\gamma^{2}\right)^{\frac{3}{2}}},
$$

which corresponds to the generalized Cauchy distribution. Furthermore, for other values of $\alpha$, the integral in (9) has no closed-form solutions; however, asymptotic series expansion exists.

Now, we consider the problem of parameter estimation of the proposed mixture model. The underlying parameter estimation based on the second-order cumulants is derived using MOLC as an approach for statistical parameter estimation different from the MOM [15].

Estimating $\alpha$ and $\gamma$ from envelope ultrasound B-mode samples is particularly important [11]. The log-cumulants of heavy-tailed $\alpha$-stable distribution which exist for all orders are given as the following [8]:

$$
\tilde{k}_{1}=\left(1-\frac{1}{\alpha}\right) \psi(1)+\ln \left(2 \gamma^{\frac{1}{\alpha}}\right)
$$

and

$$
\tilde{k}_{n}=\left[\frac{(-1)^{n}}{\alpha^{n}}+\frac{1-(-1)^{n}}{2^{n}}\right] \psi(n-1,1), n>1
$$

where $\psi(\cdot)$ is a digamma function and $\psi(n, \cdot)$ denotes a polygamma function of order $n$. Consequently, we can evaluate the characteristic exponent, $\hat{\alpha}$, as the following [25]:

$$
\hat{\alpha}=\sqrt{\frac{\hat{\tilde{k}}_{2}}{\psi(1,1)}} .
$$

Substituting $\hat{\alpha}$ in (13) and setting $n=2$, we note that the problem turns to solve the following equation:

$$
\hat{\gamma}=\exp \left\{\hat{\alpha} \hat{\tilde{k}}_{1}+\psi(1)(1-\hat{\alpha})-\hat{\alpha} \ln 2\right\} .
$$

Finally, by estimating $\hat{\alpha}$ and $\hat{\gamma}$, the corresponding mixture ratio estimate is procured.

\subsubsection{Existing approximations}

A mixture approximation for modeling the amplitude statistics of isotropic $\alpha$-stable clutter has been proposed in [26] based on the mixture ratio of $S \alpha S$. As already mentioned, isotropic $\alpha$-stable distribution is the amplitude PDF of $S \alpha S$ distribution. This mixture approximation does not provide any method to estimate the mixture ratio and it employs the traditional mixture ratio used in approximating the $S \alpha S[27,28]$, such as the logarithm moment (LM) method and the fractional lower order of moments (FLOM) method:
- LM1 method:

$$
\varepsilon_{\alpha}=2\left(\frac{\alpha-1}{\alpha}\right)
$$

- LM2 method:

$$
\varepsilon_{\alpha}=\frac{4}{3}\left(\frac{\alpha^{2}-1}{\alpha^{2}}\right)
$$

- FLOM method:

$$
\varepsilon_{\alpha}=\frac{\Gamma\left(1-\frac{p}{\alpha}\right)-\Gamma\left(1-\frac{p}{2}\right)}{\Gamma(1-p)-\Gamma\left(1-\frac{p}{2}\right)},-2<p<\alpha,
$$

where $\varepsilon_{\alpha}$ is the mixture ratio and $p$ denotes the $p$ th-order moment which is not necessarily an integer and positive. The main disadvantage of these methods is the utilization of $S \alpha S$ mixture ratio for HTR distribution, whereas the HTR distribution is the amplitude PDF of a complex $S \alpha S$ random variable and is not symmetric.

In the next section, we propose our model for amplitude PDF of ultrasound images based on a mixture approximation of HTR distribution.

\section{Proposed model}

To provide a closed-form expression for the amplitude PDF of ultrasound images which have been modeled as a HTR distribution, the generalized Cauchy-Rayleigh mixture approximation is proposed. Our proposed method constructs a new mixture for HTR distribution in the context of ultrasound image applications in which its mixture ratio contains both a characteristic exponent and a dispersion of the HTR distribution, $\alpha$ and $\gamma$. Since the $S \alpha S$ random variable representation as a scale mixture of the Gaussian random variable is traditional and therefore PDF of $S \alpha S$ can be approximated by a finite Gaussian mixture model (GMM) [29], it suggests that a similar non-Gaussian mixture model may be useful for HTR random variables. In the following, the proposed mixture approximation based on $\alpha$ and $\gamma$ with mixture ratio estimation is introduced.

\subsection{Proposed mixture approximation}

As already stated in the introduction, the LM1, LM2, and FLOM mixture ratio estimators which are employed to model the HTR distribution are only based on the $\alpha$ and they connive the role of $\gamma$ in estimating the mixture ratio, which is the main disadvantage of these methods. Furthermore, these methods are based on the moment and log-moment of $S \alpha S$ distribution, whereas the HTR distribution is the amplitude of complex $S \alpha S$ distribution which differs from traditional $S \alpha S$ distribution. Here, we propose a new mixture for modeling the amplitude PDF of ultrasound images based on 
the non-Gaussian assumption and a new version of the generalized Cauchy-Rayleigh mixture approximation, in which its mixture ratio is a function of $\alpha$ and $\gamma\left(\varepsilon_{\alpha, \gamma}\right)$. We will confine our attention to zero-mean bivariate isotropic $\alpha$-stable distribution. HTR distribution evaluation as defined in (9) requires time-consuming numerical methods to approximate the integral; however, the convergence of the integral may not be obtained because an infinite number of oscillations occurs by Bessel function. Our new mixture model is defined as the following equation:

$$
f_{\alpha, \gamma}^{\text {app }}(r)=\left(1-\varepsilon_{\alpha, \gamma}\right) f_{1, \gamma}(r)+\varepsilon_{\alpha, \gamma} f_{2, \gamma}(r) .
$$

where $\varepsilon_{\alpha, \gamma}$ is the mixture ratio and $f_{1, \gamma}(r)$ and $f_{2, \gamma}(r)$ were defined in (11) and (10), respectively. This mixture model requires only two parameters, and it can capture the algebraic tail as well as the mode. We also estimate $\varepsilon_{\alpha, \gamma}$ based on a novel approach using the HTR CF.

The PDF given in (19) can be expressed equivalently in the Fourier domain as a mixture of generalized CauchyRayleigh CF. Using (8),(9), and (19), we have,

$\exp \left(-\gamma|\omega|^{\alpha}\right)=\left(1-\varepsilon_{\alpha, \gamma}\right) \exp (-\gamma|\omega|)+\varepsilon_{\alpha, \gamma} \exp \left(-\gamma|\omega|^{2}\right)$.

It is noted that (20) is analytically characterized, and hence, mixture ratio estimation with a closed-form expression is achievable. In the following, we introduce an estimator for the mixture ratio which is basically based on the energy stored in the CF of the distribution.

\subsection{Mixture ratio estimation based on $\alpha$ and $\gamma$}

Our approach considers the equality of the energy of the distribution on both sides of (19). According to Rayleigh's (Parseval's) theorem, the total energy stored in the $r$ domain is equal to the total energy stored in the $\omega$ domain. Therefore, the energy of 2D CF is obtained as,

$$
\begin{aligned}
\int_{-\infty}^{\infty} \int_{-\infty}^{\infty}\left\|\varphi_{\alpha}(\boldsymbol{\omega})\right\|^{2} d \omega_{1} d \omega_{2}= & \int_{-\infty}^{\infty} \int_{-\infty}^{\infty} \|\left(1-\varepsilon_{\alpha, \gamma}\right) \varphi_{1}(\boldsymbol{\omega}) \\
& +\varepsilon_{\alpha, \gamma} \varphi_{2}(\boldsymbol{\omega}) \|^{2} d \omega_{1} d \omega_{2} .
\end{aligned}
$$

The right side of this equation has three terms. For simplicity, this equation can best be described as,

$$
I_{\alpha}=\left(1-\varepsilon_{\alpha, \gamma}\right)^{2} I_{1}+\varepsilon_{\alpha, \gamma}^{2} I_{2}+2 \varepsilon_{\alpha, \gamma}\left(1-\varepsilon_{\alpha, \gamma}\right) I_{12}
$$

where $I_{\alpha}, I_{1}, I_{2}$, and $I_{12}$ are the coefficients related to HTR $\mathrm{CF}$, as the following:

$$
\begin{aligned}
I_{\alpha} & =\int_{-\infty}^{\infty} \int_{-\infty}^{\infty}\left\|\varphi_{\alpha}(\boldsymbol{\omega})\right\|^{2} d \omega_{1} d \omega_{2} \\
& =\int_{-\infty}^{\infty} \int_{-\infty}^{\infty} \exp \left[-2 \gamma\left(\omega_{1}^{2}+\omega_{2}^{2}\right)^{\alpha / 2}\right] d \omega_{1} d \omega_{2},
\end{aligned}
$$

For computing the value of $I_{\alpha}$, a change of variable has been done as $\omega_{1}=\rho \cos \theta, \omega_{2}=\rho \sin \theta$ as follows:

$$
\begin{aligned}
\int_{-\infty}^{\infty} \int_{-\infty}^{\infty} \exp \left[-2 \gamma\left(\omega_{1}^{2}\right.\right. & \left.\left.+\omega_{2}^{2}\right)^{\alpha / 2}\right] d \omega_{1} d \omega_{2} \\
& =\int_{0}^{2 \pi} \int_{0}^{\infty} \rho \exp \left(-2 \gamma \rho^{\alpha}\right) d \rho d \theta
\end{aligned}
$$

using table of integrals [30], we find that

$$
\int_{0}^{\infty} x^{m} e^{-\eta x^{n}} d x=\frac{\Gamma\left(\frac{m+1}{n}\right)}{n \eta^{\frac{m+1}{n}}}, \eta>0, m>0, n>0 .
$$

The integral over $\theta$ results in $2 \pi$; hence, $I_{\alpha}$ is easily obtained as the following equation:

$$
I_{\alpha}=2 \pi \int_{0}^{\infty} \rho \exp \left(-2 \gamma \rho^{\alpha}\right) d \rho=\frac{2 \pi \Gamma\left(\frac{2}{\alpha}\right)}{\alpha(2 \gamma)^{\frac{2}{\alpha}}} .
$$

It is obvious from the above equation that the value of $I_{\alpha}$ depends on both $\alpha$ and $\gamma$. Similarly,

$$
\begin{aligned}
I_{1} & =\int_{-\infty}^{\infty} \int_{-\infty}^{\infty}\left\|\varphi_{1}(\boldsymbol{\omega})\right\|^{2} d \omega_{1} d \omega_{2} \\
& =\int_{-\infty}^{\infty} \int_{-\infty}^{\infty} \exp \left[-2 \gamma\left(\omega_{1}^{2}+\omega_{2}^{2}\right)^{\frac{1}{2}}\right] d \omega_{1} d \omega_{2} \\
& =2 \pi \int_{0}^{\infty} \rho \exp (-2 \gamma \rho) d \rho=\frac{\pi}{2 \gamma^{2}},
\end{aligned}
$$

and

$$
\begin{aligned}
I_{2} & =\int_{-\infty}^{\infty} \int_{-\infty}^{\infty}\left\|\varphi_{2}(\boldsymbol{\omega})\right\|^{2} d \omega_{1} d \omega_{2} \\
& =\int_{-\infty}^{\infty} \int_{-\infty}^{\infty} \exp \left[-2 \gamma\left(\omega_{1}^{2}+\omega_{2}^{2}\right)\right] d \omega_{1} d \omega_{2} \\
& =2 \pi \int_{0}^{\infty} \rho \exp \left(-2 \gamma \rho^{2}\right) d \rho=\frac{\pi}{2 \gamma} .
\end{aligned}
$$

$I_{1}$ and $I_{2}$ are functions of dispersion, $\gamma$. Finally, $I_{12}$ is calculated as,

$$
\begin{aligned}
I_{12} & =\int_{-\infty}^{\infty} \int_{-\infty}^{\infty} \exp \left[\gamma\left(\omega_{1}^{2}+\omega_{2}^{2}+\left(\omega_{1}^{2}+\omega_{2}^{2}\right)^{\frac{1}{2}}\right)\right] d \omega_{1} d \omega_{2} \\
& =2 \pi \int_{0}^{\infty} \rho \exp \left[-\gamma\left(\rho^{2}+\rho\right)\right] d \rho,
\end{aligned}
$$

in which an additional change of variable as $y=\rho^{2}+\rho$ is needed, so we have

$$
I_{12}=\pi \int_{0}^{\infty}\left\{\exp (-\gamma y)-\exp \left[-\gamma\left(y^{2}+y\right)\right]\right\} d y .
$$

From [30], we may write,

$$
\int_{0}^{\infty} \exp \left\{-\frac{x^{2}}{4 \xi}-\zeta x\right\} d x=\sqrt{\pi \xi} \exp \left(\xi \zeta^{2}\right)[1-\Phi(\zeta \sqrt{\xi})], \xi>0 \text {, }
$$




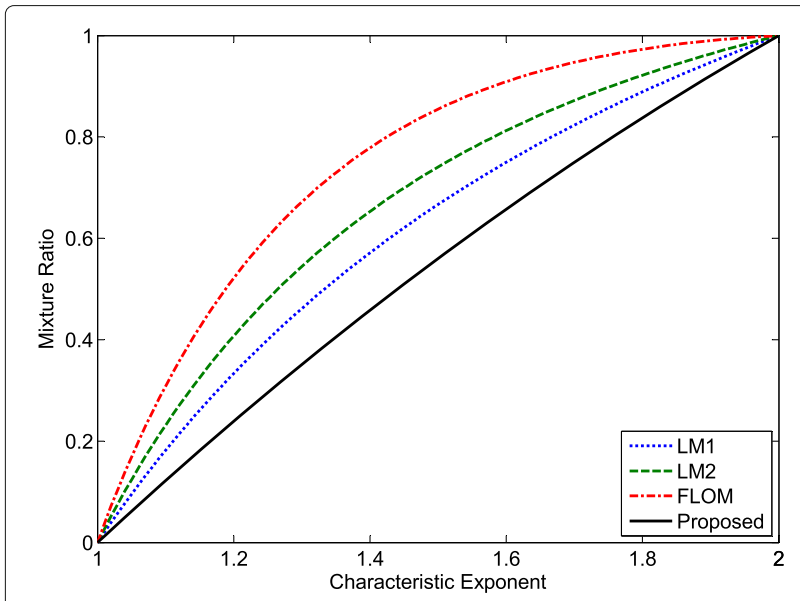

Fig. 1 Mixture ratio estimator. Different approximations of HTR distribution (9) versus values of $\alpha \in[1,2]$ with $\gamma=2, p=-1$

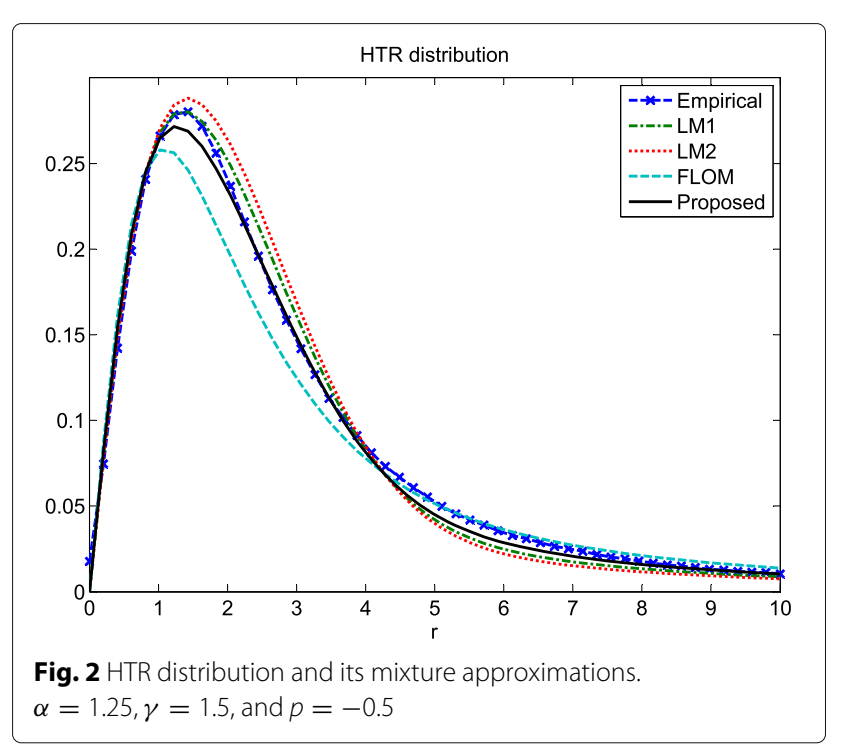

estimating the parameters, $-2<p<-0.5$ [25]. According to the simulation results, we use $p=-0.5$ as the best choice while estimating the parameters of the HTR distribution.

\subsection{Proposed mixture approximation assessment}

Now, we compare the capability of the proposed mixture with others in approximating the HTR PDF. Note that the existing approximations for the HTR distribution do not have their own mixture ratio, but they use the mixture ratio of the $S \alpha S$ distribution; in other words, they do not consider the dispersion parameter in estimating the mixture ratio. In Fig. 2, the amplitude PDF of an ideal HTR distribution (9) and its approximations based on the LM1, LM2, FLOM, and proposed mixture are depicted for $\alpha=1.25, \gamma=1.5$ with $N=$ 10,000 samples. As illustrated in this figure, the proposed mixture approximation fits more precisely the empirical HTR obtained from the samples, while sample size is small and the statistics of distribution is impulsive.

Moreover, Fig. 3a shows the approximation error between the HTR and corresponding mixture approximations for different values of $\alpha$. We used the following definition of error for evaluating the performance of the proposed method,

$$
\operatorname{error}(\mathrm{dB})=10 \log _{10}\left(\int_{0}^{\infty}\left|f_{\alpha, \gamma}(r)-f_{\alpha, \gamma}^{\mathrm{app}}(r)\right|^{2} d r\right)^{\frac{1}{2}}
$$

Even though the behavior of the samples is very impulsive due to the small values of $\alpha$, the performance of the proposed method is significantly better than the other models. Figure $3 \mathrm{~b}$ also examines the effect of order plays an important role on FLOM technique for

In this section, for quantitative evaluation of the proposed mixture, simulations in terms of error, K-S distance, K-L divergence as the goodness-of-fit tests, and computation time are provided. The appropriate choice of moment 


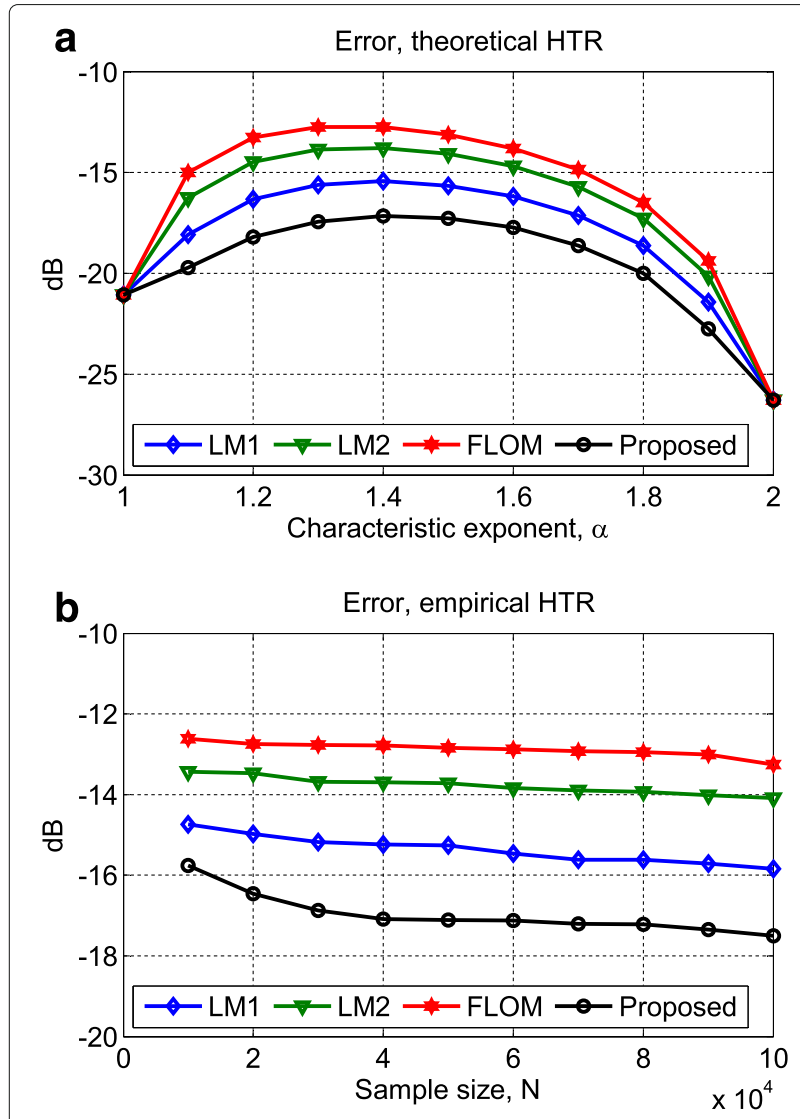

Fig. 3 Mixture approximation error. a Error between the HTR distribution and its approximations for different values of $1 \leq \alpha \leq 2$. b Error between the empirical HTR and approximations of HTR for different sample size $10,000 \leq N \leq 100,000$ and $\alpha=1.25$ (impulsive). $\gamma=1.5, p=-0.5$

sample size on the error between the empirical PDF and approximations through 1000 simulations. It is simply seen that the value of error decreases by increasing the sample size. In this figure, only different sample sizes form 10,000 to 100,000 are plotted. Since LM1, LM2, FLOM, and the proposed mixture ratios have the same value (0) for $\alpha=1$ (generalized Cauchy distribution) and the same value (1) for $\alpha=2$ (Rayleigh distribution), their performance and resultant error are the same at the beginning and at the end of Fig. 3a.

\subsection{K-S distance}

$\mathrm{K}-\mathrm{S}$ distance as a nonparametric test of the equality of continuous PDF has been successfully employed to compare a sample with a reference PDF. This statistic criterion determines a distance between the empirical cumulative distribution function (CDF) of the sample and the $\mathrm{CDF}$ of the reference distribution as the following:

$$
D_{K-S}=\sup _{r}\left\|F_{\alpha, \gamma}(r)-F_{\alpha, \gamma}^{\mathrm{app}}(r)\right\|,
$$

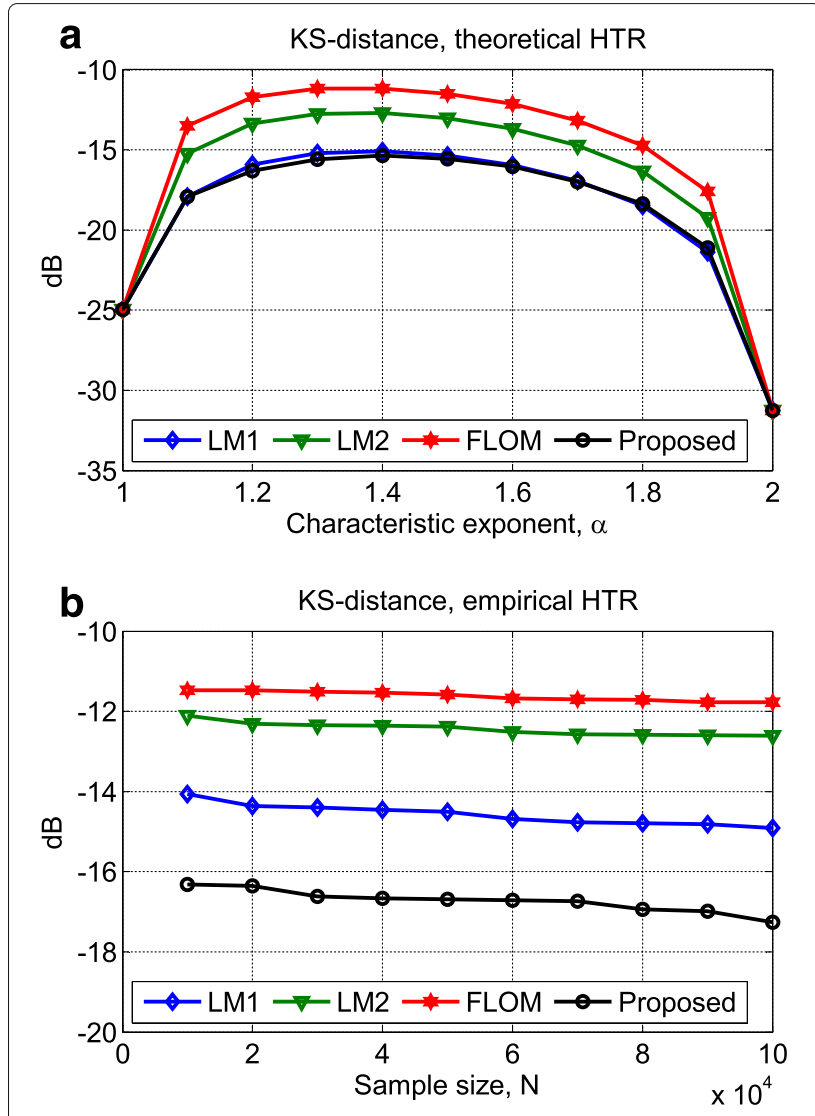

Fig. 4 K-S distance as a goodness-of-fit test. a K-S distance between the approximations and theoretical HTR, $1 \leq \alpha \leq 2$. b K-S distance between the approximations and empirical HTR,

$10,000 \leq N \leq 100,000$ and $\alpha=1.25 \cdot \gamma=1.5, p=-0.5$

where sup is the supremum and $F_{\alpha, \gamma}(\cdot)$ and $F_{\alpha, \gamma}^{\text {app }}(\cdot)$ denote the empirical CDF and the CDF computed from the approximated PDF, respectively. The K-S distance computed for the true HTR distribution and its four approximations is depicted in Fig. 4a based on different characteristic exponents from impulsive $(\alpha=1)$ to smooth $(\alpha=2)$ statistics, $\gamma=1.5$. We carried out 1000 simulations to estimate the K-S test similar to the previous section for synthetic samples of a HTR distribution with $\alpha=1.25, \gamma=1.5$ and for $10,000 \leq N \leq 100,000$, and the simulation results are demonstrated in Fig. $4 \mathrm{~b}$. In this way, as discussed before, the performances of the mixture estimators is identical for $\alpha=1$ and $\alpha=2$, while at the middle of the region $(\alpha=1.5)$, the lowest efficiency appears.

\subsection{Computation time}

Now, the performance of the HTR mixture ratio estimators is verified in the context of time complexity and in terms of execution time on a machine. 
Table 1 Mean computation time (in milliseconds) obtained from the 1000 simulation runs for the HTR mixture approximations based on $\alpha=1.5$ and $\gamma=3$

\begin{tabular}{lllll}
\hline & LM1 & LM2 & FLOM & Proposed \\
\hline$N=10^{3}$ & 0.58 & 0.62 & 0.65 & 0.78 \\
$N=10^{4}$ & 0.90 & 0.94 & 0.95 & 0.99 \\
$N=10^{5}$ & 5.15 & 5.29 & 5.61 & 5.95 \\
$N=10^{6}$ & 52.1 & 53.0 & 54.6 & 59.3 \\
\hline
\end{tabular}

To compare the computation time of LM1, LM2, FLOM, and the proposed method, the number of samples $(N)$ plays an important role. On the other hand, the algorithm used to estimate the parameters of the HTR distribution is the same for all four aforementioned methods, so this step imposes a constant burden on all estimators. LM1 uses one addition and the number of its multiplications is two, whereas LM2 requires one addition and five multiplications. The number of additions and multiplications for FLOM are five and three, respectively. Moreover, a computation of a gamma built-in function is needed. The complexity of our estimator in terms of the number of multiplications is $O(1)$. The computation time in the proposed method is directly dependent on the calculation of exponential and error function. In addition to the above discussion, the computation time of the estimators is examined by performing 1000 simulation runs. The mean computation time for four mixture ratio estimators of the HTR distribution based on the proposed and the LM1,
LM2, and FLOM methods is illustrated in Table 1 for different values of $N=10^{3}, 10^{4}, 10^{5}, 10^{6}$ and $\alpha=$ $1.5, \gamma=3$. All the 1000 simulations are performed on a PC machine with 64-bit operating system and Intel Core i5-2430M CPU and 4-GB RAM under MATLAB 2012a. As predicted, the computation time of our estimator is greater than the other methods, and the LM1 method has the smallest computational burden. On the other hand, since the proposed estimator considers both $\alpha$ and $\gamma$ in estimating the mixture ratio, it is efficient in the context of accuracy and goodness of fit.

\section{Experiments on real ultrasound images}

The congruence of the proposed mixture approximation based on the HTR distribution as a non-Gaussian statistical tool for modeling the amplitude statistics of medical ultrasound images is experimentally scrutinized. For experimental assessment, we used four rectangle B-mode ultrasound images: (1) common carotid artery (CCA) in longitudinal section; (2) thyroid cyst, internal calcification; (3) pancreas in tissue harmonic mode; and (4) breast mass. This dataset is provided by the SPLab research group of the Brno University of Technology and the images taken from Samsung Medison ultrasound scanners. In this study, we consider only ultrasound images; however, the results in this paper are general and can be used to model other types of medical images [31]. The resolution of images is $300 \times$ 300 pixels. The ultrasound images used in the simulations are shown in Fig. 5. Using the MOLC estimator,
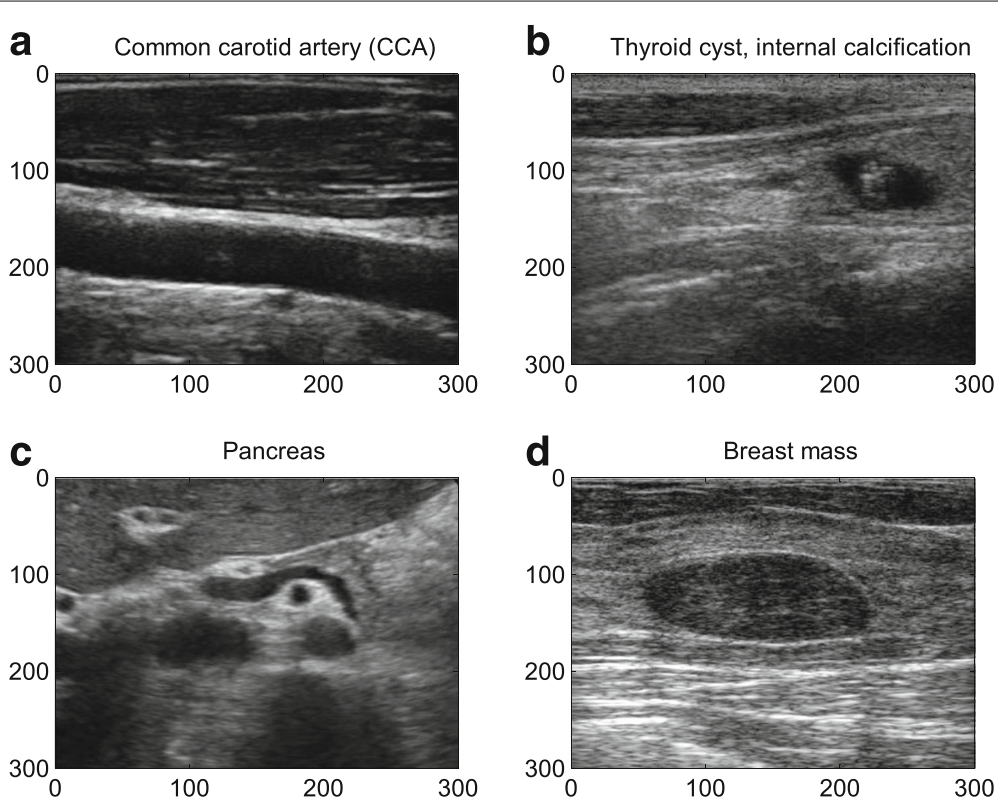

Fig. 5 Dataset. a Common carotid artery (CCA) in longitudinal section; b thyroid cyst, internal calcification; c the pancreas in tissue harmonic mode; d breast mass. CCA image is provided by the SPLab research group of the Brno University of Technology. The other three ultrasound images are provided by Samsung Medison ultrasound scanners 


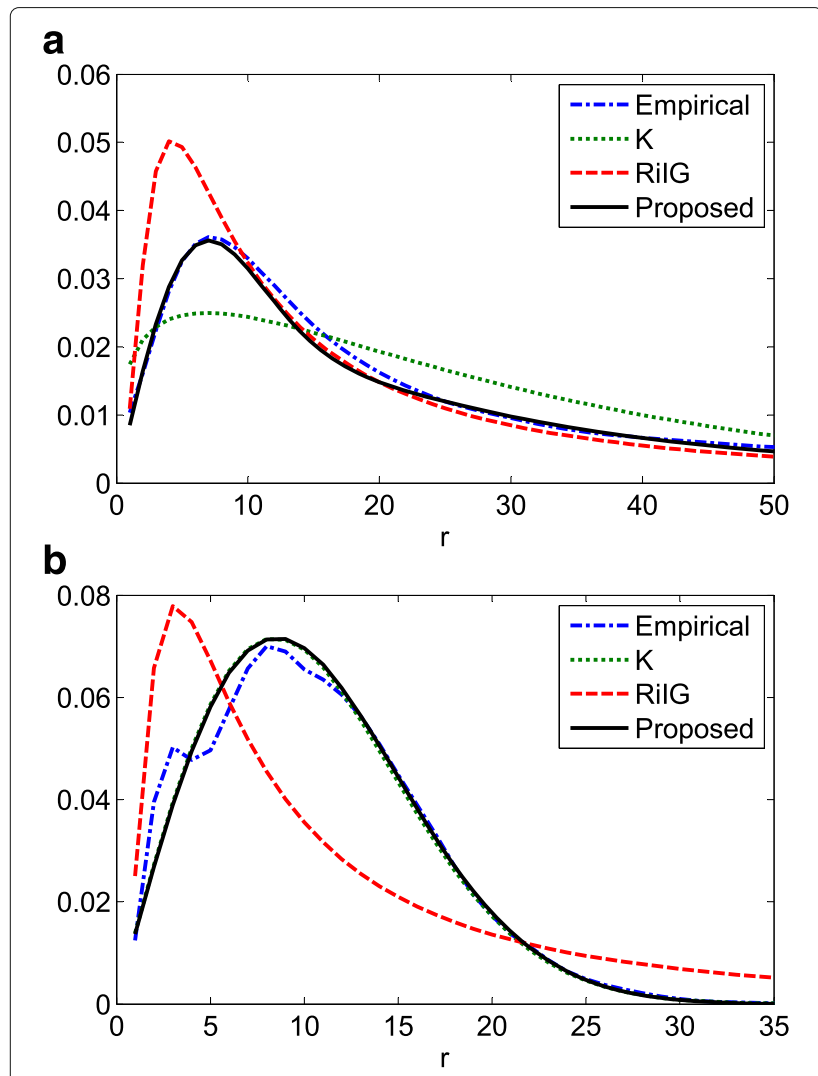

Fig. 6 Comparison of the amplitude PDF obtained from the K, RilG, and HTR-proposed distributions. Ultrasound images: $\mathbf{a}$ CCA and $\mathbf{b}$ thyroid cyst, internal calcification we estimate the parameters of the HTR distribution: $\alpha=1.2042, \gamma=18.8584$ for the CCA ultrasound image; $\alpha=1.9505, \gamma=36.5655$ for the thyroid cyst ultrasound image; $\alpha=1.8160, \gamma=98.9915$ for the pancreas ultrasound image; and $\alpha=1.6939, \gamma=40.7420$ for the breast mass ultrasound image. Also, the parameter estimation based on MOLC for the K model has been, done and we obtained $v=[-0.3089,0.4786,1.6197,1.2159]$ and $\sigma=$ [ 25.8518, 55.9408, 38.7482, 28.3202] for four ultrasound images. Parameter estimation of the RiIG model with $a_{0}=2$ and $\delta_{0}=20$ is performed based on IMLM. For CCA, $a=3.0394, \lambda=0.1725, \delta=1.9891$; the thyroid cyst, $a=3.2811, \lambda=0.4372, \delta=1.2166$; the pancreas, $a=4.4463, \lambda=0.4095, \delta=1.3952$; and the breast mass, $a=3.5398, \lambda=0.4302, \delta=$ 1.2472 are calculated. The experimental results depicted in Fig. 6a give a comparison between our proposed scheme for amplitude distribution and previously studied amplitude distributions for the image taken from CCA. Figure $6 \mathrm{~b}$ also illustrates that the envelope of signals backscattered from thyroid cyst is best defined by the HTR model using proposed mixture approximation

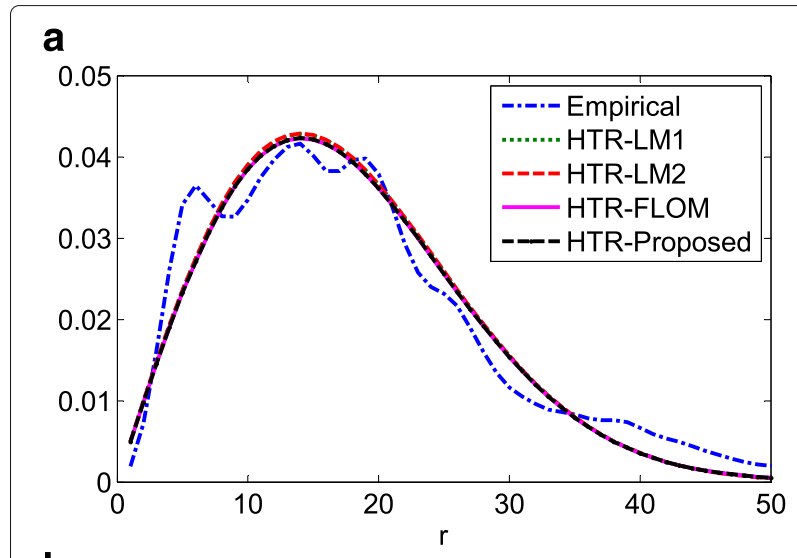

b

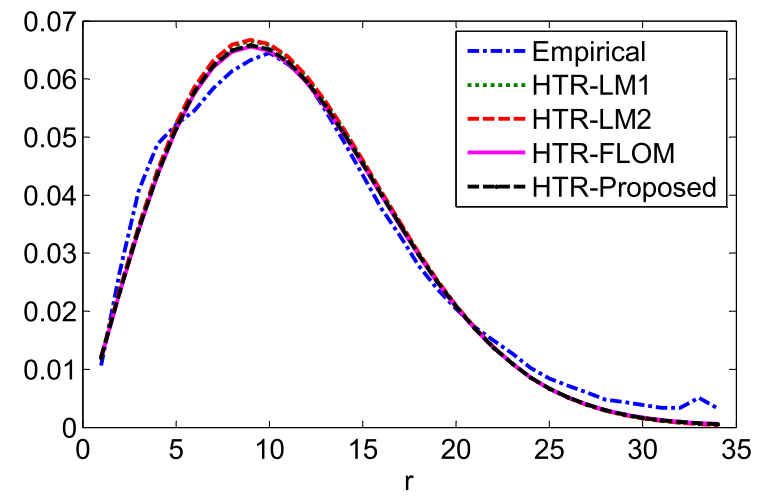

Fig. 7 Experimental results on different mixture ratio approximations. This figure demonstrates the results of experiments for amplitude PDF of ultrasound images: $\mathbf{a}$ the pancreas in tissue harmonic mode and $\mathbf{b}$ breast mass. Comparison is done using the HTR-LM1, HTR-LM2, HTR-FLOM, and HTR-proposed approximations

ratio. Moreover, from Fig. 7a, b, it can be apperceived that the proposed mixture approximation precisely fits the empirical PDF and the tail of the empirical data with respect to those of the HTR-LM1, HTR-LM2, and HTR-FLOM mixture approximation models for ultrasound images taken from the pancreas and breast mass, respectively. We find that the HTR-proposed distributions provide a better fitness than the K, RiIG, HTRLM1, HTR-LM2, and HTR-FLOM distributions. It is very important to note that the RiIG model has three parameters, whereas the HTR and K models have two parameters.

Table 2 displays error for different models used for ultrasound images. However, when compared to other amplitude PDF estimation methods in ultrasound imagery, the HTR-proposed approach has a lower error. The errors of the proposed method for ultrasound images are $4.3804,0.1298,0.0185$, and $0.2388 \mathrm{~dB}$ lower than the best error obtained based on the other methods. In Table 3, the parameter estimation for the six models 
Table 2 Comparison of the six models in the context of error (dB)

\begin{tabular}{lllllll}
\hline & K & RilG & HTR-LM1 & HTR-LM2 & HTR-FLOM & HTR-Proposed \\
\hline CCA & -14.7025 & -12.7357 & -14.6616 & -12.9651 & -13.6374 & -19.0420 \\
Thyroid cyst & -16.6479 & -13.8617 & -16.7142 & -16.7285 & -16.6356 & -16.8583 \\
Pancreas & -16.1737 & -11.5753 & -16.2033 & -16.1832 & -16.2709 & -16.2894 \\
Breast mass & -18.3238 & -10.2990 & -18.0846 & -18.0010 & -18.3902 & -18.6290 \\
\hline
\end{tabular}

used for the PDF estimation of ultrasound images are also presented, together with their respective $\mathrm{K}$ $\mathrm{S}$ distances. The HTR-proposed distribution has the least and the HTR-LM2 and RiIG models have the worst K-S distance. The K-distribution and HTR-FLOM have the closest K-S distances to the proposed model. The comparison of the HTR-proposed model with the K-distribution shows a better efficiency of 8.33, $11.91,11.79$, and $2.60 \%$ for ultrasound images $1-4$, respectively.

\subsection{K-L divergence}

In the following, we present the experimental results obtained from the K-L divergence test by our proposed method with the ones obtained by K, RiIG, HTR-LM1, HTR-LM2, and HTR-FLOM. For distributions $f_{\alpha, \gamma}(r)$ and $f_{\alpha, \gamma}^{\text {app }}(r)$ of a continuous random variable, the K-L divergence or $\mathrm{K}-\mathrm{L}$ distance is defined to be the following integral:

$$
D_{K-L}\left(f_{\alpha, \gamma}(r) \| f_{\alpha, \gamma}^{\mathrm{app}}(r)\right)=\int_{0}^{\infty} f_{\alpha, \gamma}(r) \ln \frac{f_{\alpha, \gamma}(r)}{f_{\alpha, \gamma}^{\mathrm{app}}(r)} d r
$$

where $f_{\alpha, \gamma}(r)$ denotes the empirical PDF. The above equation is a measurement of the difference between two probabilities; particularly, it denotes the information lost when $f_{\alpha, \gamma}^{\text {app }}(r)$ is used to approximate $f_{\alpha, \gamma}(r)$. Table 4 depicts that the HTR-proposed model has the lowest value and it improves the best goodness of fitness obtained with other methods by $22.8,86.7,2.9$, and $1.8 \%$ in the context of the $\mathrm{K}-\mathrm{L}$ divergence criterion.

\section{$5.2 L_{m}$-Norm}

Finally, in order to investigate the validity of the error criterion, we further perform simulations to measure the $L_{m}$-norm between the PDF estimated by different models and empirical PDF obtained from real ultrasound data. For a real number $m>1, L_{m}$-norm is defined by,

Table 3 K-S distance and parameter estimation for K, RilG, HTR-LM1, HTR-LM2, HTR-FLOM, and HTR-proposed models

\begin{tabular}{|c|c|c|c|c|c|c|c|c|c|}
\hline \multirow{2}{*}{ Model } & \multirow{2}{*}{ Parameters } & \multicolumn{2}{|c|}{ CCA } & \multicolumn{2}{|c|}{ Thyroid cyst } & \multicolumn{2}{|c|}{ Pancreas } & \multicolumn{2}{|c|}{ Breast mass } \\
\hline & & & $\mathrm{K}-\mathrm{S}$ dis & & K-S dis & & K-S dis & & K-S dis \\
\hline \multirow{3}{*}{ K } & $\hat{v}$ & -0.3089 & \multirow{3}{*}{0.0732} & 0.4786 & \multirow{3}{*}{0.0227} & 1.6197 & \multirow{3}{*}{0.0343} & 1.2159 & \multirow{3}{*}{0.0172} \\
\hline & $\hat{\sigma}$ & 25.8518 & & 55.9408 & & 38.7482 & & 28.3202 & \\
\hline & $\hat{a}$ & 3.0394 & & 3.2811 & & 4.4463 & & 3.5398 & \\
\hline \multirow[t]{3}{*}{ RilG } & $\hat{\lambda}$ & 0.1725 & \multirow[t]{3}{*}{0.1349} & 0.4372 & \multirow[t]{3}{*}{0.1893} & 0.4095 & \multirow[t]{3}{*}{0.1538} & 0.4302 & \multirow[t]{3}{*}{0.1709} \\
\hline & $\hat{\delta}$ & 1.9891 & & 1.2166 & & 1.3952 & & 1.2472 & \\
\hline & $\hat{\alpha}$ & 1.2042 & & 1.9505 & & 1.8160 & & 1.6939 & \\
\hline \multirow[t]{3}{*}{ HTR-LM1 } & $\hat{\gamma}$ & 18.8584 & \multirow[t]{3}{*}{0.0982} & 36.5655 & \multirow[t]{3}{*}{0.0231} & 98.9915 & \multirow[t]{3}{*}{0.0369} & 40.7420 & \multirow[t]{3}{*}{0.0213} \\
\hline & $\hat{\varepsilon}_{\alpha}$ & 0.3392 & & 0.9548 & & 0.8327 & & 0.6568 & \\
\hline & $\hat{\alpha}$ & 1.2042 & & 1.9505 & & 1.8160 & & 1.6939 & \\
\hline \multirow[t]{3}{*}{ HTR-LM2 } & $\hat{\gamma}$ & 18.8584 & \multirow[t]{3}{*}{0.1517} & 36.5655 & \multirow[t]{3}{*}{0.0232} & 98.9915 & \multirow[t]{3}{*}{0.0391} & 40.7420 & \multirow[t]{3}{*}{0.0241} \\
\hline & $\hat{\varepsilon}_{\alpha}$ & 0.4139 & & 0.9532 & & 0.8167 & & 0.6333 & \\
\hline & $\hat{\alpha}$ & 1.2042 & & 1.9505 & & 1.8160 & & 1.6939 & \\
\hline \multirow[t]{3}{*}{ HTR-FLOM } & $\hat{\gamma}$ & 18.8584 & \multirow[t]{3}{*}{0.1446} & 36.5655 & \multirow[t]{3}{*}{0.0225} & 98.9915 & \multirow[t]{3}{*}{0.0272} & 40.7420 & \multirow[t]{3}{*}{0.0200} \\
\hline & $\hat{\varepsilon}_{\alpha}$ & 0.0155 & & 0.9623 & & 0.8420 & & 0.6649 & \\
\hline & $\hat{\alpha}$ & 1.2042 & & 1.9505 & & 1.8160 & & 1.6939 & \\
\hline \multirow[t]{2}{*}{ HTR-Proposed } & $\hat{\gamma}$ & 18.8584 & \multirow[t]{2}{*}{0.0671} & 36.5655 & \multirow[t]{2}{*}{0.0217} & 98.9915 & \multirow[t]{2}{*}{0.0272} & 40.7420 & 0.0169 \\
\hline & $\hat{\varepsilon}_{\alpha, \gamma}$ & 0.2169 & & 0.9603 & & 0.8053 & & 0.6288 & \\
\hline
\end{tabular}


Table 4 Numerical results obtained from K-L divergence test for four ultrasound images

\begin{tabular}{llllllr}
\hline & K & RilG & HTR-LM1 & HTR-LM2 & HTR-FLOM & HTR-Proposed \\
\hline CCA & 0.1041 & 0.1328 & 0.0994 & 0.1296 & 0.1315 & 0.0767 \\
Thyroid cyst & 0.0068 & 0.2974 & 0.0033 & 0.0044 & -0.0015 & -0.0002 \\
Pancreas & 0.0497 & 0.2243 & 0.0431 & 0.0419 & 0.0499 & 0.0407 \\
Breast mass & 0.0215 & 0.2671 & 0.0249 & 0.0230 & 0.0246 & 0.0211 \\
\hline
\end{tabular}

$$
\left\|f_{\alpha, \gamma}(r)-f_{\alpha, \gamma}^{\mathrm{app}}(r)\right\|_{m}=\left(\int_{0}^{\infty}\left|f_{\alpha, \gamma}(r)-f_{\alpha, \gamma}^{\mathrm{app}}(r)\right|^{m} d r\right)^{\frac{1}{m}} .
$$

We apply the proposed parametric method for mixture ratio estimation of the CCA ultrasound image. In Fig. 8, the simulation results for the six different techniques are given. It can be deduced from this figure that the proposed method has better consistency with the different-distance criterion defined as $L_{m}$-norm. Comparing the $L_{m}$-norms, we realized that the value of error remains approximately constant for $m>5$. Numerical results verify that the developed model has the superior performance and the RiIG model has the worst performance for amplitude PDF estimation.

\section{Conclusions}

In this paper, an effective scheme for statistically nonGaussian modeling of the amplitude PDF of ultrasound images is proposed. The basic idea of the model is to represent a mixture approximation for HTR distribution with a closed-form formula whose mixture ratio is a function of both $\alpha$ and $\gamma$. In particular, we not only consider the characteristic exponent on estimating the mixture ratio but also the influence of dispersion has been investigated. Performance comparison between the proposed method and the existing ones is adopted through several

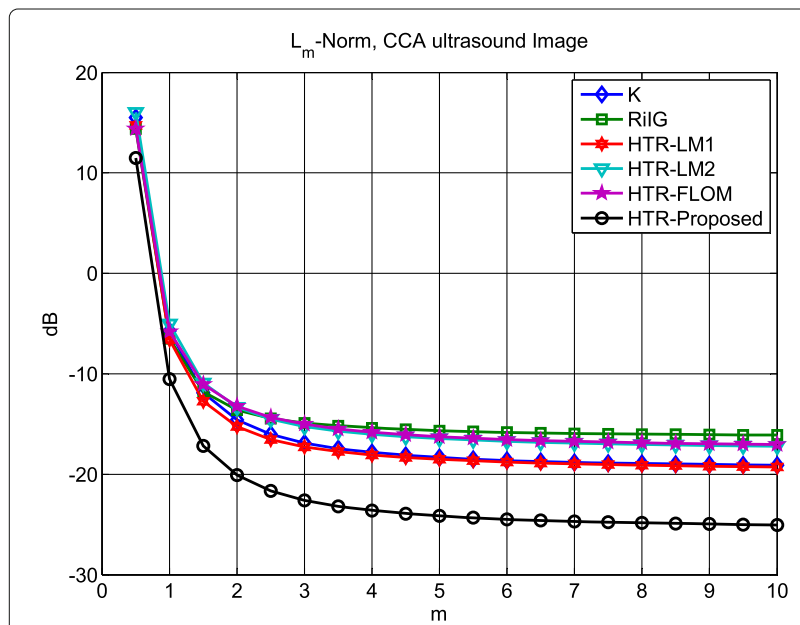

Fig. $8 \mathrm{Lm}$-Norm. CCA ultrasound image: $\hat{\alpha}=1.2042, \hat{\gamma}=18.8584$ simulations, and the experimental results obtained from real ultrasound images in terms of error, K-S distance, K$\mathrm{L}$ divergence, and $L_{m}$-norm confirm the plausibility of the new approach in terms of accuracy and fitness.

\section{Authors' contributions \\ MHB elaborated the novel generalized Cauchy-Rayleigh mixture, carried out the experiments and statistical analysis of the images, and participated in drafting the manuscript. HA participated in the conception and design of the study, offered useful suggestions, and helped to draft the manuscript and revise it critically for important intellectual content. Both authors read and approved the final manuscript.}

\section{Competing interests}

The authors declare that they have no competing interests.

Received: 24 May 2016 Accepted: 20 November 2016

Published online: 19 December 2016

\section{References}

1. SHC Ortiz, T Chiu, MD Fox, Ultrasound image enhancement: a review. Biomed. Signal Process. Control. 7(5), 419-428 (2012)

2. F Varray, C Cachard, A Ramalli, P Tortoli, O Basset, Simulation of ultrasound nonlinear propagation on GPU using a generalized angular spectrum method. EURASIP J. Image Video Process. 2011(1), 1-6 (2011)

3. HD Cheng, J Shan, W Ju, Y Guo, L Zhang, Automated breast cancer detection and classification using ultrasound images: a survey. Pattern Recog. 43(1), 299-317 (2010)

4. F Destrempes, M-HR Cardinal, L Allard, J-C Tardif, G Cloutier, Segmentation method of intravascular ultrasound images of human coronary arteries. Comput. Med. Imaging Graph. 38(2), 91-103 (2014)

5. N Afsham, M Najafi, P Abolmaesumi, R Rohling, A generalized correlation-based model for out-of-plane motion estimation in freehand ultrasound. Med. Imaging IEEE Trans. 33(1), 186-199 (2014)

6. V Damerjian, O Tankyevych, N Souag, E Petit, Speckle characterization methods in ultrasound images - a review. IRBM. 35(4), 202-213 (2014)

7. F Destrempes, G Cloutier, A critical review and uniformized representation of statistical distributions modeling the ultrasound echo envelope. Ultrasound Med. Biol. 36(7), 1037-1051 (2010)

8. A Achim, A Basarab, G Tzagkarakis, P Tsakalides, D Kouame, Reconstruction of ultrasound RF echoes modeled as stable random variables. Comput. Imaging IEEE Trans. 1(2), 86-95 (2015)

9. THiguchi, S Hirata, T Yamaguchi, H Hachiya, Liver tissue characterization for each pixel in ultrasound image using multi-Rayleigh model. Jpn. J. Appl. Phys. 53(7S), 07-27 (2014)

10. RW Prager, AH Gee, GM Treece, LH Berman, Decompression and speckle detection for ultrasound images using the homodyned k-distribution. Pattern Recog. Lett. 24(4-5), 705-713 (2003)

11. P Mohana Shankar, VA Dumane, JM Reid, V Genis, F Forsberg, CW Piccoli, BB Goldberg, Classification of ultrasonic B-mode images of breast masses using Nakagami distribution. Ultrason. Ferroelectr. Freq. Control IEEE Trans. 48(2), 569-580 (2001)

12. A Gupta, et al, Study of compound generalized Nakagami-generalized inverse Gaussian distribution and related densities: application to ultrasound imaging. Comput. Stat. 30(1), 81-96 (2015)

13. M Pereyra, N Dobigeon, H Batatia, J-Y Tourneret, in Signal Processing Conference, 2011 19th European. Segmentation of ultrasound images 
using a spatially coherent generalized Rayleigh mixture model (IEEE, 2011), pp. 664-668

14. Q Peng, L Zhao, SAR image filtering based on the Cauchy-Rayleigh mixture model. Geosci. Remote Sens. Lett. IEEE. 11(5), 960-964 (2014)

15. SN Anfinsen, T Eltoft, Application of the matrix-variate Mellin transform to analysis of polarimetric radar images. Geosci. Remote Sens. IEEE Trans. 49(6), 2281-2295 (2011)

16. KShanmugam, R Wahidabanu, Condensed anisotropic diffusion for speckle reducton and enhancement in ultrasonography. EURASIP J. Image Video Process. 2012(1), 1-17 (2012)

17. TEltoft, Modeling the amplitude statistics of ultrasonic images. Med. Imaging IEEE Trans. 25(2), 229-240 (2006)

18. TEltoft, The Rician inverse Gaussian distribution: a new model for non-Rayleigh signal amplitude statistics. Image Process. IEEE Trans. 14(11), 1722-1735 (2005)

19. DW Kim, C Kim, DH Kim, DH Lim, Rician nonlocal means denoising for MR images using nonparametric principal component analysis. EURASIP J. Image Video Process. 2011(1), 1-8 (2011)

20. L Weng, JM Reid, PM Shankar, K Soetanto, Ultrasound speckle analysis based on the K distribution. J. Acoust. Soc. Am. 89(6), 2992-2995 (1991)

21. V Krylov, G Moser, SB Serpico, J Zerubia, Modeling the statistics of high resolution SAR images. Research Report RR-6722, INRIA (2008). https://hal. inria.fr/inria-00342681/file/RR-6722.pdf

22. CL Nikias, M Shao, Signal Processing with $\alpha$-Stable Distributions and Applications. (Wiley, New York, 1995)

23. M Pereyra, $\mathrm{H}$ Batatia, Modeling ultrasound echoes in skin tissues using symmetric alpha-stable processes. Ultrason. Ferroelectr. Freq. Control IEEE Trans. 59(1), 60-72 (2012)

24. M Pereyra, N Dobigeon, H Batatia, J Tourneret, Segmentation of skin lesions in 2-D and 3-D ultrasound images using a spatially coherent generalized Rayleigh mixture model. Med. Imaging IEEE Trans. 31(8), 1509-1520 (2012)

25. EE Kuruoglu, J Zerubia, Modeling SAR images with a generalization of the Rayleigh distribution. Image Process. IEEE Trans. 13, 527-533 (2004)

26. X Li, S Wang, L Fan, X Lei, Mixture approximation to the amplitude statistics of isotropic $\alpha$-stable clutter. Signal Process. 99, 86-91 (2014)

27. $\mathrm{XMa}, \mathrm{CL}$ Nikias, Parameter estimation and blind channel identification in impulsive signal environments. IEEE Trans. Signal Process. 43(12), 2884-2897 (1995)

28. $X T$ Li, J Sun, LW Jin, M Liu, Bi-parameter CGM model for approximation of $\alpha$-stable PDF. Electron. Lett. 44(18), 1096-1097 (2008)

29. A Swami, in Acoustics, Speech, and Signal Processing, 2000. ICASSP' '00. Proceedings. 2000 IEEE International Conference On. Non-Gaussian mixture models for detection and estimation in heavy-tailed noise, vol. 6 (IEEE, 2000), pp. 3802-3805

30. IS Gradshteyn, IM Ryzhik, Table of Integrals, Series, and Products, 7th edn. (Academic Press, Elsevier Science, 2007)

31. M Pereyra, N Dobigeon, H Batatia, J-Y Tourneret, in 2011 IEEE International Conference on Acoustics, Speech and Signal Processing (ICASSP). Labeling skin tissues in ultrasound images using a generalized Rayleigh mixture model (IEEE, 2011), pp. 729-732

\section{Submit your manuscript to a SpringerOpen ${ }^{\circ}$ journal and benefit from:}

- Convenient online submission

- Rigorous peer review

- Immediate publication on acceptance

- Open access: articles freely available online

- High visibility within the field

- Retaining the copyright to your article

Submit your next manuscript at $\boldsymbol{s p r i n g e r o p e n . c o m ~}$ 